\title{
Costo-efectividad de la resincronización biventricular como alternativa de tratamiento de la insuficiencia cardiaca
}

\author{
Cost-effectiveness of the biventricular resynchronization as an \\ alternative therapy for heart failure
}

\author{
Antonio Castillo Guzmán'; Roberto Zayas Molina'"; Carmen Arocha \\ Mariño"l'; Nurys B. Armas Rojas ${ }^{\prime V}$; Digna Camejo Esquijarosav \\ 'Máster en Ciencias de la Salud. Profesor Auxiliar. Instituto de Cardiología y Cirugía \\ Cardiovascular. La Habana, Cuba. \\ "Doctor en Ciencias. Profesor Titular. Instituto de Cardiología y Cirugía \\ Cardiovascular. La Habana, Cuba. \\ "IIDoctora en Ciencias de la Salud. Profesora Titular. Escuela Nacional de Salud \\ Pública. La Habana, Cuba. \\ IVEspecialista de II Grado en Epidemiología. Profesora Auxiliar. Instituto de \\ Cardiología y Cirugía Cardiovascular. La Habana, Cuba.

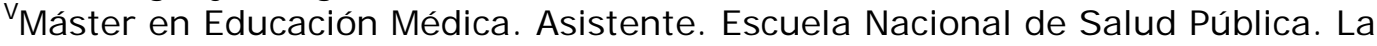 \\ Habana, Cuba.
}

\section{RESUMEN}

Introducción La evolución final de la mayoría de las enfermedades cardiovasculares es el síndrome de insuficiencia cardiaca, su tratamiento puede abordarse de diferentes formas. Para aquellos pacientes refractarios a tratamiento farmacológico, uno de los más recientemente utilizados es la resincronización biventricular.

Objetivos Identificar los costos y el grado de efectividad de la resincronización biventricular como una opción en el tratamiento de enfermos con insuficiencia cardiaca refractaria y bloqueo de la rama izquierda del haz de His.

Métodos Se realizó una evaluación económica del tipo costo-efectividad según la clasificación de Drummond y otros. Fue considerado un horizonte temporal que osciló entre seis meses y cinco años. Las alternativas consideradas fueron: la resincronización biventricular y la terapia farmacológica como alternativa teórica de la no resincronización. El universo lo integraron 30 enfermos con insuficiencia 
cardiaca refractaria a tratamiento. La variable de efectividad considerada fue: muerte evitada.

Resultados El $76,6 \%$ de los enfermos eran del sexo masculino y el $23,4 \%$ del sexo femenino. La mortalidad fue de $36,6 \%$ en el período de seguimiento. La efectividad del proceder alcanzó el 63,4\%, con 19 muertes evitadas. El costo efectividad incremental fue de 33 805,00 pesos. Conclusiones La introducción en Cuba de la alternativa de tratamiento de la insuficiencia cardiaca, conocida como resincronización biventricular resulta costo-efectiva.

Palabras clave: Costo-efectividad, resincronización biventricular, síndrome de insuficiencia cardiaca.

\section{ABSTRACT}

Introduction The final evolution of most of cardiovascular diseases is the heart failure syndrome and there are several ways to treat it. For those patients refractory to drug therapy, one of the most recent methods is biventricular resyncronization.

Objectives To identify costs and level of effectiveness of biventricular resynchronization as a choice of treatment of refractory heart failure patients with His' bundle left branch blocking.

Methods A cost-effectiveness type economic assessment was made following Drummond et al's classification. The considered period of time was 6 months to one year. The analyzed alternatives were biventricular resynchronization and drug therapy as theoretical alternative to non-resynchronization. The universe of study was 30 patients suffering heart failure refractory to treatment. The effectiveness variable was prevented death.

Results In this group, $76.6 \%$ of patients were males and $23.4 \%$ were females. Mortality rate was $36.6 \%$ in the follow-up period. The effectiveness of the procedure was $63.4 \%$ representing 19 prevented deaths. The incremental costeffectiveness was $\$ 33805.00$ pesos.

Conclusions The introduction of therapeutic option known as biventricular resynchronization to treat heart failure was cost-effective.

Key words: Cost-effectiveness, biventricular resynchronization, heart failure syndrome.

\section{NTRODUCCI ÓN}

La evaluación económica constituye un método cada vez más utilizado para la toma de decisiones ante alternativas u opciones en el sector de la salud pública. ${ }^{1,2}$

El tratamiento de la primera causa de muerte en Cuba y en el mundo, las enfermedades cardiovasculares, tiene un amplio abanico de opciones que obliga no sólo a los terapeutas, sino también a administradores, políticos y a los propios 
clientes, usuarios o pacientes a considerar las posibilidades económicas antes de optar por una alternativa.

La evolución final de la mayoría de estas enfermedades es el síndrome de insuficiencia cardiaca. Por otro lado, aunque resulte paradójico, un mejor control de los factores de riesgo que favorecen su aparición, como la hipertensión arterial y el infarto del miocardio, entre otros, han incrementado la esperanza de vida y han elevado, a su vez, la morbilidad y la mortalidad por esta causa. ${ }^{3,4}$

La introducción de agentes vasodilatadores, especialmente los inhibidores de la enzima convertidora de la angiotensina II, los betabloqueadores, los diuréticos de ASA y la digoxina han demostrado mejoría en la capacidad funcional de los pacientes con enfermedades cardiovasculares (The Consensos 1987, ${ }^{5}$ The SOLVD $1991^{6}$ ), no obstante, un considerable número de ellos llega a la etapa más avanzada e irreversible de insuficiencia cardiaca, en la que los fármacos no son suficientes para mantener una capacidad funcional aceptable y existe una amenaza para la vida.

El empleo de dispositivos de asistencia circulatoria mecánica como la contrapulsación aórtica, el hemopums, la bomba centrífuga, la bomba Axipum, el dispositivo DeBakey/NASA, la bomba rotatoria a flujo pulsátil desarrollada en China y el corazón artificial eléctrico, ${ }^{7,8}$ han evitado fallos de bomba agudos, o, en el mejor de los casos, han servido de puente para el transplante cardiaco.

Para los casos más avanzados de insuficiencia cardiaca, el transplante de corazón es el tratamiento de elección, encontrándose este proceder, actualmente, en su grado de madurez, pero con distintas restricciones como pueden ser: número reducido de donantes para un cada vez mayor número de receptores, las contraindicaciones clínicas, las limitaciones logísticas, psicológicas, biológicas y las de índole sociocultural, de modo tal que es aplicable a un reducido número de pacientes.

Los enfermos a los que no se les puede realizar el trasplante cardiaco forman el sustrato sobre los que se ha utilizado distintas alternativas con el propósito de lograr una mejoría de la función ventricular sistólica y diastólica entre ellas la miocardioplastia dinámica, ${ }^{9}$ la miocardioplastia celular ${ }^{10}$ y la resincronización biventricular. ${ }^{11}$

En Cuba se han practicado las tres opciones; la primera se abandonó a principios de los años 90 por falta de presupuesto a causa del efecto negativo sobre la economía producido por el derrumbe del campo socialista y por el recrudecimiento del bloqueo económico impuesto por los Estados Unidos. Hoy en día la técnica de la miocardioplastia dinámica está en desuso.

En el caso de la miocardioplastia celular, los resultados obtenidos en Cuba son parciales, toda vez que el número de pacientes a los que se les practicó esta alternativa es muy reducido y a nivel mundial, aún se considera en etapa de investigación. ${ }^{12}$

El propósito de esta investigación es identificar los costos y el grado de efectividad de la resincronización ventricular como una opción en el tratamiento de pacientes con insuficiencia cardiaca refractaria y bloqueo de la rama izquierda del haz de His. Los resultados obtenidos servirán a la dirección del Instituto de Cardiología y Cirugía Cardiovascular (ICCCV) para solicitar a las autoridades del Ministerio de 
Salud Pública (MINSAP) los fondos necesarios para incrementar su aplicación dada la alta prevalencia de esta afección.

\section{MÉTODOS}

Se realizó una evaluación económica del tipo costo-efectividad según la clasificación de Drummond y otros. ${ }^{13}$ Se consideró como horizonte temporal un periodo que osciló entre 6 meses y 5 años.

Las alternativas consideradas fueron: la resincronización ventricular y la no resincronización ventricular (teórica).

El universo lo integraron 30 pacientes con insuficiencia cardiaca congestiva refractaria a fármacos, clase funcional III-IV de la New York Heart Asociation ${ }^{14}$ y bloqueo de la rama izquierda del haz de His que acudieron al servicio de arritmología y estimulación cardiaca del ICCCV entre el primero de marzo de 1999 y el 30 de noviembre del 2003. La insuficiencia cardiaca fue considerada refractaria cuando los síntomas y signos persistían a pesar del uso adecuado de fármacos de primera línea (digitálicos, diuréticos, inhibidores de la enzima convertidora de la angiotensina, betabloqueadores y los antagonistas de los receptores de la angiotensina II).

Para la alternativa teórica de la no resincronización ventricular se decidió que un número igual de pacientes (30) recibirían sólo tratamiento medicamentoso hospitalizados ( 12 días en terapia intensiva y 7 días en la sala), para ello se utilizó el criterio de expertos del ICCCV. No se incluyeron pacientes que pudieran tener posibles complicaciones. Como mortalidad para este grupo, se tomó el 23,9\% según lo encontrado en el estudio COMPANION. ${ }^{15}$

No se incluyeron los costos indirectos ocasionados por las erogaciones de bolsillo y días dejados de trabajar por los pacientes y familiares. Se realizó una estimación de los costos incrementales y no se hizo un análisis de sensibilidad por considerar robustas las variables estudiadas.

\section{RESULTADOS}

El $76,6 \%$ de los pacientes fueron del sexo masculino y el $23,4 \%$ del sexo femenino. Las edades comprendidas entre los 51 y 60 años agruparon a la mayoría de los pacientes con el $46,6 \%$ del total.

El $50 \%$ de la muestra tenía una miocardiopatía isquémica con antecedentes de un infarto cardiaco y el 43,3\% padecía una miocardiopatía dilatada de causa desconocida.

La mortalidad fue de 36,6 \% (11 fallecidos) en el período de seguimiento que osciló entre 6 meses y 3 años.

Según el horizonte temporal previsto, la resincronización ventricular evitó 19 muertes para el $63,4 \%$ de efectividad (figura). 
No. de casos

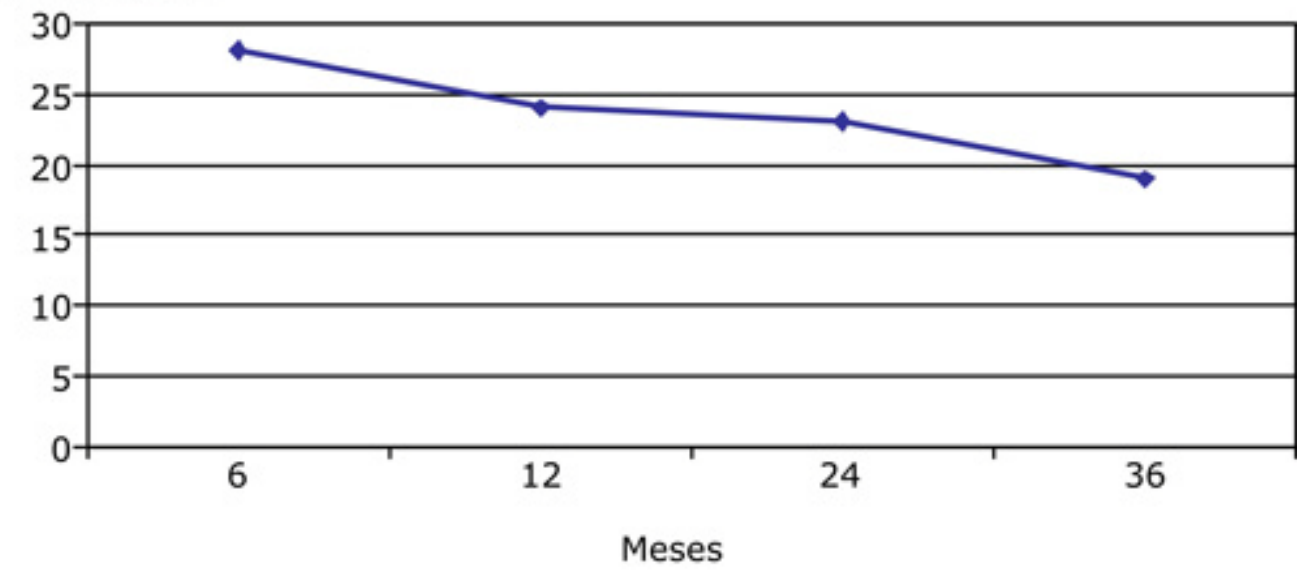

Fig. 1. Curva de Supervivencia (Kaplan-Meier) en pacientes con resincronización ventricular ICCCV 1999-2003.

El costo total de la resincronización ventricular fue de 205470,43 pesos, con una media de 9348,77 ( $\pm 2553,21$ ). Dos componentes, los marcapasos y la estadía hospitalaria total representaron el $63,34 \%$ el primero y el 33,65 \% el segundo, del costo total (tabla 1).

Tabla 1. Costo de la estimulación biventricular según sus componentes

\begin{tabular}{|c|c|c|c|c|}
\hline \multirow{2}{*}{$\begin{array}{l}\text { Componentes de los } \\
\text { costos }\end{array}$} & \multirow[t]{2}{*}{ Costo total } & \multicolumn{2}{|c|}{ Costo en pesos* } & \multirow{2}{*}{$\begin{array}{l}\text { Desviación } \\
\text { estándar }( \pm \text { ) }\end{array}$} \\
\hline & & $\%$ & Media & \\
\hline Estadía total & 69148,00 & 33,65 & 2881,17 & 2453,54 \\
\hline $\begin{array}{l}\text { Medicamentos y } \\
\text { hemoderivados }\end{array}$ & 847,32 & 0,41 & 35,31 & 19,44 \\
\hline Investigaciones & 1989,32 & 0,97 & 82,89 & 18,46 \\
\hline Material gastable & 1933,76 & 0,94 & 80,57 & 7,41 \\
\hline Marcapasos & 130140,93 & 63,34 & 5422,54 & 1788,59 \\
\hline Salón OP & 1411,10 & 0,69 & 58,80 & 12,21 \\
\hline Total & 205470,43 & 100,00 & 9348,77 & 2553,21 \\
\hline
\end{tabular}

* Se consideró el cambio: 1 dólar estadounidense = 1 peso cubano.

El costo total de la alternativa teórica fue de 70250,4 pesos, para un solo ingreso al año (tabla 2). El 94,9 \% del costo total correspondió a la estadía en la unidad de terapia más la estadía en la sala. La tabla 3 muestra que el costo-efectividad incremental fue de 33805,0 pesos por cada muerte evitada. 
Tabla 2. Costo del tratamiento en pacientes hospitalizados (alternativa teórica) por insuficiencia cardiaca según sus componentes

\begin{tabular}{|l|c|c|c|}
\hline \multirow{2}{*}{ Componentes de los costos } & \multicolumn{2}{|c|}{ Costo en pesos } \\
\cline { 2 - 3 } & Costo total & $\%$ & Media \\
\hline Estadia total & 66704,4 & 94,9 & 2223,5 \\
\hline Medicamentos y hemoderivados & 1059,3 & 1,5 & 35,3 \\
\hline Investigaciones & 2486,7 & 3,6 & 82,9 \\
\hline Total & 70250,4 & 100,0 & 2341,7 \\
\hline
\end{tabular}

Tabla 3. Costo-efectividad por alternativa

\begin{tabular}{|l|c|c|}
\hline Costo / Efectividad & Resincronización miocárdica & Alternativa teórica \\
\hline Costo total & 205470,4 & 70250,4 \\
\hline Efectividad (muertes evitadas) & 19 & 15 \\
\hline Costo/efectividad & 10814,2 & 4683,4 \\
\hline Costo/efectividad (incremental) & 33805,0 & - \\
\hline
\end{tabular}

\section{DISCUSIÓN}

No hay muchas investigaciones publicadas que hagan un análisis de los costos que origina la terapia de resincronización ventricular.

La supervivencia alcanzada en esta investigación, a los 36 meses de seguimiento, fue de $63,4 \%$. Hernández $\mathrm{A}$ y otros ${ }^{16}$ registran el $90 \%$ en un seguimiento medio de 15,3 $3 \pm 9,45$ meses. Los períodos de seguimiento no son iguales por lo que no es correcto hacer comparaciones. Algunos autores como García-Boloo I y otros ${ }^{17}$ y Alberca MT junto a García-Cosío $F^{18}$ opinan que son necesarios estudios aleatorizados con seguimientos a largo plazo para evaluar la supervivencia.

El estudio COMPANION ${ }^{15}$ no solamente comparó los resultados de la resincronización ventricular con un grupo que utilizó únicamente terapia medicamentosa, sino también con el uso de los desfibriladores automáticos implantables (DAI) encontrándose que con este último la mortalidad se reducía en el $36 \%$ contra el $23,9 \%$ con la resincronización ventricular sola, al año; pero ambos mostraron un mejor pronóstico que con la terapia farmacológica (alternativa teórica en esta investigación). De cualquier modo, la disminución de la mortalidad alcanzada en este estudio, con una supervivencia de 79,2 y $63,3 \%$ al año y a los tres años justifica el empleo de esta terapia. 
En el costo promedio de $9348,77( \pm 2553,21)$ pesos, los marcapasos aportaron el $58 \%$ y la estadía el $30,8 \%$, de modo que, al no poder reducirse el precio de los primeros, lo lógico sería pensar en disminuir la estadía para abaratar el costo total, pero un gran número de estos pacientes necesitan primero mejorar su estado clínico antes de ser sometidos a la terapia de resincronización, por lo tanto, no es probable que la estadía pueda reducirse en forma importante, no obstante, si se compara con el costo por egreso anual de cada paciente con insuficiencia cardiaca en Estados Unidos que es de 10000 dólares, ${ }^{19}$ y que, el pronóstico de los pacientes sin resincronización, refractarios al tratamiento farmacológico, necesitarán no sólo un incremento farmacológico, siempre que lo toleren, sino que el número de ingresos anuales crecerá, demandando una mayor utilización de las unidades de terapia intensiva, ese costo inicial que parece significativo se amortiza con la reducción de la hospitalización. Hernández $\mathrm{A}$ y otros encuentran que el costo inicial que supone la implantación del dispositivo se amortiza a los 14,6 meses. ${ }^{16}$

El costo-efectividad incremental encontrado mostró que cada muerte evitada por insuficiencia cardiaca tuvo un costo de 33805,0 pesos, cifra que no resulta alta si se tiene en cuenta que la vida humana no tiene precio.

La terapia de resincronización ventricular no sólo es un medio de tratamiento de la insuficiencia cardiaca refractaria a medicamentos, sino que también sirve de puente para un posible trasplante cardiaco cuando esté disponible un órgano. ${ }^{20}$

La introducción de la alternativa conocida como resincronización ventricular constituye una terapia efectiva para el tratamiento de la insuficiencia cardiaca refractaria a medicamentos. Su elevado costo no significa un impedimento para que el estado cubano disponga del presupuesto necesario para el tratamiento de los pacientes necesitados. La existencia de una red cardiológica nacional debe facilitar su generalización a todo el país.

\section{REFERENCI AS BI BLI OGRÁFiCAS}

1. Gálvez AM, Álvarez M, Saduy Y, Morales M. Diagnóstico de la evaluación económica en salud en Cuba. Rev Cubana Salud Pública. 2004;30(1): 8-16.

2. Baly A. Toledo ME, Rodríguez F. La economía de la salud, la eficiencia y el costo de oportunidad. Rev Cubana Med Gen Integr. 2001;17(4):395-8.

3. Lopera G, Castellanos A, de Marchena E. Nuevos fármacos en insuficiencia cardiaca. Rev Esp Cardiol. 2001; 54:624-34.

4. Céspedes LA, Castañar JF, Gómez R. Insuficiencia cardiaca. En: Álvarez Sintes R, editor científico. Temas de Medicina General Integral. La Habana. Editorial Ciencias Médicas; 2001.p.556-68.

5. The CONSENSUS trial study group. Effect of enalapril on mortality in severe congestive heart failure: results of Cooperative North Scandinavian Enalapril Suavival Study (CONSENSUS). N Engl J Med. 1987;316:1429-35.

6. The SOLVD Investigators. Effect of enalapril on survival in patients with reduced lefty ventricular ejection fractions and congestive heart failure. N Engl J Med. 1991; 325: 293-302. 
7. Rowles JR, Khanwilkar PS, Diegel PD, Hansen AC, Bearnson GB, Smith KD, et al. Development of a totally implantable artificial heart. ASAIO J. 1992;38:713-6.

8. Mussivaud T, Hendry PJ, Masters RG, Holmes KS, Hum A, Keon WJ. A remotely controlled and powered artificial heart pump. Artificial Org. 1996;20(12):1314-9.

9. Dumcios A, Chekanov V, Vysockas V. Current status of cardiomyoplatyas current alternative for end-stage heart failure. Medicina. 2003; 39:815-22.

10. Hérnandez A, Hidalgo J. La regeneración del corazón y la terapia celular con células madre. Revista CENIC. 2004;5(3): 210-1.

11. Zayas R. Resincronización ventricular como tratamiento de la insuficiencia cardiaca refractaria con bloqueo de rama izquierda [tesis]. La Habana: Instituto de Cardiología y Cirugía Cardiovascular; 2005.

12. Nadal-Ginard B, Torella D, Ellison G. Medicina regenerativa cardiovascular en la encrucijada. Es urgente basar los ensayos clínicos sobre terapia celular en datos sólidos obtenidos en animales experimentales relevantes para los humanos. Rev Esp Cardiol. 2006; 59:1175-89.

13. Drummond MF, O'Brien BJ, Stodart GL, Torrance GW. Métodos para la Evaluación Económica de los Programas de Asistencia Sanitaria. 2da ed. Madrid: Díaz de Santos; 2001.

14. Morales D, Fajuri A. Temas de Medicina Interna. Insuficiencia cardiaca, tratamiento médico[sitio en Internet]. [citada 3 Ene 2007]. Disponible en: http://escuela. med.puc.cl/paginas/publicaciones/temasmedicinainterna/icc. html

15. Bristow MR, Saxon LA, Boehmer J, Krueger S, Kass DA, De Marco T, et al. Comparison of Medical Therapy, PACINE, and Defibrillation in Heart Failure. (COMPANION) Investigators. Cardiac-resynchronization therapy with or without an implantable defibrillator in advanced chronic heart failure. N Engl J Med. 2004; 350: 2140-50.

16. Hernández A, Escobar C, Marín I, Bernal E. Resincronización cardiaca. Impacto socioeconómico sanitario. Mortalidad del procedimiento. Relación coste-beneficios. Impacto en la clase funcional/ calidad de vida. Pronóstico y seguimiento. Investigación Cardiovascular. 2005; 8(2):135-50.

17. García Boloo I, Macías A, Alegría E, Berengel A, Gavira JJ, Azcárate P, et al. Tratamiento de la insuficiencia cardiaca avanzada mediante estimulación biventricular. Experiencia inicial en una serie de 22 casos consecutivos. Res Esp Cardiol. 2003; 56:245-52.

18. Alberca MT, García-Cosío F. Resincronización ventricular en la insuficiencia cardiaca: ¿un método bien establecido o una línea de progreso con muchas interrogantes? Rev Esp Cardiol. 2003; 56:637-41.

19. O'Connel JB. The economic burden of heart failure. Clin Cardiol. 2000;23 (suppl. III): III-6. 
20. Greenbg J M, Lion AL, Book WM, Hot BJ, DeLurgio DB, Langberg JJ, et al. Benefits of cardiac resynchronization therapy in outpatients with indicators of heart transplantation. J Heart Lung Transp. 2003;22:1134-40.

Recibido: 8 de febrero de 2008.

Aprobado: 22 de octubre de 2008.

Antonio Castillo Guzmán. Instituto de Cardiología y Cirugía Cardiovascular (ICCCV). La Habana, Cuba.

E-mail acastillo@icccv.sld.cu 